\title{
EXPORTACIÓN Y PERSPECTIVA DEL COMERCIO DE LA PALTA HASS PERUANA
}

\author{
PERSPECTIVE EXPORT AND TRADE PERUVIAN HASS AVOCADO
}

\author{
Bartolomé Jorge Anyosa Gutiérrez '
}

\begin{abstract}
RESUMEN
En esta investigación presentamos cuadros sobre la evolución de la producción y el comercio de la palta Hass Peruana en el mercado interno, desde el año 1990 y las exportaciones registradas oficialmente, desde 2004, por la Superintendencia Nacional deAduanas del Perú.

Nos hemos basado en métodos de matrices de impacto cruzado y el análisis morfológico para trabajar con el equilibrio de poder entre los actores y un gran número de variables cualitativas que mantienen relación de alta dependencia entre ellos. Se construyeron cuatro escenarios alternativos, en base a estimaciones de crecimiento económico, crecimiento poblacional, consumo de la palta y organizaciones internacionales, con los cuales se hicieron predicciones cuantitativas de este mercado.
\end{abstract}

Palabras clave: perspectiva, escenarios, hegemonía, predicciones.

\begin{abstract}
The purpose of the research is to present the forecast of the exportation of the "Hass" avocado in the international market and the participation of the Peruvian production in the domestic market.

For this construction, there were used methods of cross-impact matrices and morphologic analysis, which let us work with the power equilibrium between the performers and with a great number of qualitative variables that kept a high dependence relation between them. There were constructed four alternative scenarios: in basis of forecasting of economic growth, population growth, avocado consumption and international organizations, which were used to make quantitative forecasts of this market.
\end{abstract}

Keywords: perspective, scenarios, hegemony, forecasts.

\section{INTRODUCCIÓN.}

La producción de la palta Hass en el Perú pretende tener una importancia real en el futuro. La operación comercial con el mercado externo y los saldos a obtener de esta operación permitió incrementar la reserva nacional, mejorar la economía y brindar mayor bienestar social a la población peruana y así resistir la crisis económica mundial.

El análisis de las variables del sector de producción y exportación revela cuáles están en crecimiento y cuáles no. Esta situación permite conocer la ubicación de los mercados y las condiciones de la demanda de palta para el consumo y, de igual manera, los bienes de capital que tanta falta hace para el desarrollo productivo de las empresas instaladas en nuestro país.

El Estado dispone de un Plan de desarrollo Nacional, con el eje temático Competitividad del País y el Ministerio de Comercio Exterior y Turismo, con el Plan Nacional de Exportaciones, los cuales permiten analizar los principales mercados internacionales y poner en conocimiento de los productores los cambios que suceden y según esos indicadores elaborar productos exclusivos en calidad y sanidad para los mercados exigentes. 


\section{Objetivos}

Evaluar las predicciones en el mercado internacional de la palta Hass.

Seleccionar los principales mercados internacionales consumidores de palta Hass.

\section{LA PALTA SUS CARACTERÍSTICAS Y PRODUCCIÓN.}

La palta o aguacate es el fruto de un árbol originario de México y Centroamérica. Antes de la llegada de los españoles su cultivo se extendía hasta lo que es ahora Colombia, Ecuador y Perú.

El nombre aguacate, con el que se le conoce en muchos países de habla hispana, es una adaptación de ahuacatl, vocablo de origen náhuatl, lengua Maya.

El árbol del aguacate actualmente se cultiva en numerosas regiones de clima tropical o templado como México, Indonesia, Estados Unidos, República Dominicana, Brasil, Israel, Chile, Perú, Sudáfrica, entre otros. Existen diversas variedades de aguacate, entre ellas la Hass, Bacon, Ettinger, Fuerte, Nabal, Reed, Ryan y Wurtz.

El aguacate o palta es la más completa de las frutas y verduras, de gran valor alimenticio, contiene todas las vitaminas del reino vegetal (A, $\mathrm{B}, \mathrm{C}, \mathrm{D}, \mathrm{E}, \mathrm{K}$ ), minerales (potasio, manganeso, magnesio, hierro y fósforo), y proteínas. Algunos utilizan el fruto, las hojas y la semilla en medicina natural para combatir problemas del aparato digestivo; y las hojas como expectorante.
El Perú tiene un área productora de palta de aproximadamente 17500 Hectáreas de las cuales aproximadamente 9000 son de variedad Hass, 3 ooo Hectáreas de fuerte y el resto de una mezcla de variedades caracterizadas por su bajo contenido de aceite.

Las zonas productivas son: La Libertad, Áncash, Lima, Ica, Arequipa, Ayacucho, Moquegua y Tacna. Curiosamente existe una marcada diferenciación entre las zonas productoras de Palta Hass y Fuerte (Zona costera, desértica) con las otras variedades que se producen en zonas cálidas y lluviosas próximas a la amazonia.

En el año 1994 se inician las primeras plantaciones de Hass llevadas a cabo por empresarios agricultores con el propósito de exportar. Perú exporta casi exclusivamente a Europa, del total, el $20 \%$ va al mercado Inglés, el $40 \%$ a Francia y el $40 \%$ a España. El $95 \%$ de la palta que se exporta es Hass y el $5 \%$ restante corresponde a Etinger y Fuerte.

El área de producción de este cultivo abarca la mayoría de la costa central del País.

Los principales valles productivos se ubican en Trujillo, Áncash, Casma, Barranca, Huacho, Chancay, Huaral, Cañete e Ica. La temporada de la palta Hass se encuentra disponible desde Marzo hasta Junio. Los meses de exportación más frecuentes van entre enero y setiembre. Sin embargo, los picos de envíos altos ocurren en marzo, abril y mayo.

Tabla $\mathbf{N}^{\circ}$ o1: Superficie, Volumen y Rendimiento de Palta Hass en el Perú

\begin{tabular}{|l|r|r|r|r|r|r|r|r|r|r|}
\hline & $\mathbf{2 0 0 1}$ & $\mathbf{2 0 0 2}$ & $\mathbf{2 0 0 3}$ & $\mathbf{2 0 0 4}$ & $\mathbf{2 0 0 5}$ & $\mathbf{2 0 0 6}$ & $\mathbf{2 0 0 7}$ & $\mathbf{2 0 0 8}$ & $\mathbf{2 0 0 9}$ & $\mathbf{2 0 1 0}$ \\
\hline Superficie Cultivada (Miles de ha) & 13,7 & 14,4 & 15,1 & 16,0 & 16,5 & 18,1 & 20,5 & 22,3 & 27,0 & 31,2 \\
\hline Volumen de Producción (miles de t) & 93,5 & 94,2 & 100,0 & 108,5 & 103,4 & 113,3 & 121,7 & 136,3 & 157,4 & 184,4 \\
\hline Rendimiento Promedio (t/ha) & 13,7 & 14,4 & 15,1 & 16,0 & 16,5 & 18,1 & 20,5 & 22,3 & 27,0 & 31,2 \\
\hline
\end{tabular}

Fuente: ProHass PERU

Elaboración: Propia

Tabla $\mathbf{N}^{\circ}$ 02: Exportación en tonelada y valor en dólares de Palta Hass en el Perú.

\begin{tabular}{|c|c|c|c|c|c|c|c|c|c|c|}
\hline & 2001 & 2002 & 2003 & 2004 & 2005 & 2006 & 2007 & 2008 & 2009 & 2010 \\
\hline (Miles de US\$ FOB) & 3478 & 4926 & 15722 & 18708 & 23367 & 38802 & 47343 & 70818 & 64393 & 84638 \\
\hline (Miles de t) & 2,5 & 4,8 & 11,5 & 14,6 & 18,7 & 31,7 & 37,5 & 51,3 & 48,3 & 59,5 \\
\hline (Precio por $\mathrm{t}$ ) & 1391 & 1026 & 1367 & 1281 & 1250 & 1224 & 1262 & 1380 & 1393 & 1422 \\
\hline
\end{tabular}

Fuente: ProHass PERU

Elaboración: Propia 
Tabla $N^{\circ}$ 03: Principales Países Productores de Palta Hass en el mundo.

\begin{tabular}{|c|c|c|c|c|c|c|c|c|c|c|}
\hline Países & 2001 & 2002 & 2003 & 2004 & 2005 & 2006 & 2007 & 2008 & 2009 & 2010 \\
\hline Mundo & 398,293 & 644,876 & 615,644 & 917,991 & 847,405 & $1,318,343$ & $1,398,268$ & $1,488,949$ & $1,461,784$ & $2,012,750$ \\
\hline México & 102,606 & 195,063 & 211,255 & 366,123 & 329,272 & 601,177 & 577,199 & 645,412 & 594,010 & 887,642 \\
\hline Chile & 70,318 & 181,674 & 199,495 & 171,251 & 122,128 & 229,581 & 156,494 & 263,668 & 188,542 & 226,346 \\
\hline Perú & 4,872 & 15,792 & 18,708 & 23,367 & 38,793 & 46,812 & 72,840 & 64,393 & 84,848 & 164,399 \\
\hline Holanda & 21,764 & 28,356 & 34,600 & 46,272 & 61,570 & 85,611 & 111,619 & 125,361 & 126,741 & 167,089 \\
\hline España & 51,121 & 63,117 & 87,777 & 81,591 & 96,489 & 99,154 & 144,938 & 121,408 & 115,448 & 169,668 \\
\hline Israel & 42,703 & 34,000 & 0 & 58,525 & 41,901 & 60,417 & 42,480 & 54,412 & 75,040 & 71,487 \\
\hline Sudáfrica & 17,701 & 23,552 & 21,237 & 34,816 & 18,737 & 27,847 & 31,712 & 24,777 & 43,663 & 30,737 \\
\hline Kenya & 1,788 & 10,927 & 9,718 & 11,180 & 9,642 & 13,223 & 17,485 & 20,793 & 21,718 & 24,897 \\
\hline N. Zelandia & 12,018 & 15,539 & 19,658 & 28,801 & 26,927 & 35,359 & 39,212 & 34,395 & 37,791 & 67,690 \\
\hline R. Dominicana & 10,599 & 8,723 & 11,522 & 12,704 & 11,399 & 16,389 & 21,385 & 16,281 & 17,991 & 21,326 \\
\hline
\end{tabular}

Fuente: FAOSTAT

Elaboración: Propia

Tabla $\mathrm{N}^{\circ}$ 04: Principales países consumidores de Palta Hass en el mundo.

\begin{tabular}{|c|c|c|c|c|c|c|c|c|c|c|}
\hline Países & 2001 & 2002 & 2003 & 2004 & 2005 & 2006 & 2007 & 2008 & 2009 & 2010 \\
\hline $\mathrm{USA}$ & 108,522 & 134,425 & 137,844 & 259,080 & 186,142 & 339,291 & 296,357 & 420,126 & 316,308 & 386,803 \\
\hline Francia & 79,906 & 75,418 & 90,411 & 88,260 & 75,194 & 84,077 & 74,111 & 80,631 & 93,520 & 118,352 \\
\hline Japón & 13,648 & 23,974 & 28,991 & 28,150 & 29,032 & 26,511 & 24,073 & 29,840 & 44,552 & 64,455 \\
\hline Canadá & 14,988 & 15,879 & 19,143 & 18,243 & 21,876 & 23,252 & 25,083 & 28,610 & 35,646 & 47,356 \\
\hline Países Bajos & 11,759 & 8,159 & 10,232 & 14,425 & 11,015 & 13,075 & 6,882 & 16,209 & 29,947 & $39,4,79$ \\
\hline Italia & 0 & 2,588 & 3,233 & 3,284 & 3,606 & 3,922 & 0 & 21,582 & 24,685 & 28,468 \\
\hline Alemania & 11,205 & 11,976 & 12,795 & 13,573 & 12,990 & 16,691 & 13,323 & 14,680 & 20,990 & 26,509 \\
\hline Suecia & 5,648 & 4,984 & 7,618 & 8,738 & 6,500 & 8,392 & 11,764 & 12,466 & 14,170 & 17,370 \\
\hline Dinamarca & 4,066 & 3,908 & 4,823 & 5,687 & 6,262 & 6,923 & 7,264 & 9,245 & 11,382 & 13,826 \\
\hline Reino Unido & 28,229 & 25,885 & 29,069 & 56,661 & 57,978 & 42,145 & 37,877 & 21,293 & 11,374 & 10,749 \\
\hline
\end{tabular}

\section{Fuente: FAOSTAT}

Elaboración: Propia

\subsection{Lugar de estudio}

El equipo de estudio se instaló en la ciudad de Tacna. Se visitó al experto en producción y comercialización de palta Hass (PROHASS) en la ciudad de Lima, con quien de realizó las coordinaciones sobre el trabajo de investigación. Para conocer el volumen exportado de palta Hass. Los datos se recabaron de la Superintendencia Nacional de Aduanas del Perú, y para la validación de de los instrumentos se operó con el apoyo de un Maestro en Administración de Negocios, experto en prospectiva.

\subsection{Metodología}

En la investigación se ha construido algunos escenarios para el sector de la palta Hass de Perú, en base a variables cualitativas y al diseño de la producción y venta de dichos productos en el mundo, así como la participación de la producción peruana. Hemos visto los escenarios futuros relacionados entre sí, que involucran a múltiples actores. Existe una fuerza que puede determinar la evolución del mercado. Definimos el "comercio internacional de palta Hass producido en Perú" como un sistema, que sufre el efecto de muchas variables y la acción de diversos actores, que tratan de analizar estos aspectos para entender y predecir sus movimientos en diferentes situaciones.

Para trabajar con este conjunto de variables y actores se han utilizado una serie de herramientas desarrolladas por Michel Godet en LIPSOR Laboratorio de Investigación en Prospectiva, Estrategia y Organización. Estas herramientas están en algoritmos simples, utilizan la multiplicación de la matriz, y ayudan en la selección de las variables y actores en los sistemas complejos, así como la construcción de escenarios. Las herramientas se centraron en la planificación estratégica y han demostrado su utilidad para el análisis que hemos propuesto en este trabajo. 


\subsection{Análisis estructural de variables relevantes}

\section{Análisis Estructural Prospectivo Método Micmac}

En la proyección de escenarios futuros para la palta Hass es necesario entender, no sólo la acción de las variables que influyen en ellos, sino también la relación de dependencia entre las mismas, ya que puedan capturar los efectos indirectos que cada uno causa al otro.

$\mathrm{El}$ análisis estructural es una herramienta que a través del uso de un software, permite estructurar una reflexión colectiva, mediante la posibilidad de describir un sistema a partir de una matriz que relaciona todos los elementos que la constituyen.

La Tabla $\mathrm{N}^{\circ} 05$ presenta la lista de variables que consideramos las más relevantes para el futuro del mercado internacional de la palta Hass producido en Perú y en esencia, representan las variables cualitativas.

El siguiente paso del análisis consistió en la construcción de una matriz cuadrada con todas estas variables (líneas "i" y columnas "j"), rellena con un valor que representa la influencia de la variable "i" a la variable "j". Dado el carácter integral de las variables, se optó por una escala de valoración reducido donde:

\begin{tabular}{cl} 
Valor & Grado de Influencia \\
\hline $0:$ & Ninguno \\
$1:$ & Bajo \\
$2:$ & Alta
\end{tabular}

La suma de los valores asignados a cada fila determina el grado de influencia de una variable sobre la otra, que llamamos motor, mientras que la suma de los valores de cada columna representan el grado de su dependencia de otras variables.

Tabla $\mathbf{N}^{\circ}$ 05: Listado de variables.

\begin{tabular}{|c|c|c|}
\hline $\mathrm{N}^{\circ}$ & Título largo & Título corto \\
\hline 1 & La tasa de crecimiento de la economía mundial. & TASACRESC \\
\hline 2 & La tasa de crecimiento del comercio mundial. & TASACOM \\
\hline 3 & Las diferencias hegemonía mundial. & HEGEMUN \\
\hline 4 & La capacidad de actuación de los Estados Nacionales. & ESTADO \\
\hline 5 & Las negociaciones multilaterales de comercio. & $\mathrm{OMC}$ \\
\hline 6 & La negociación comercial con USA. & TLC \\
\hline 7 & Las negociaciones al interior de la Comunidad Andina de Naciones. & CAN \\
\hline 8 & La producción de palta en el mundo y la capacidad de consumo de palta. & PRODMUNDO \\
\hline 9 & El precio de la palta. & PRECIOPALT \\
\hline 10 & El precio de otras variedades de palta. & PREVAPALTA \\
\hline 11 & La política para fomentar la producción de palta. & PRODPALTA \\
\hline 12 & La política nacional agrícola. & POLAGRI \\
\hline 13 & La política interna de consumo de palta. & POLCONS \\
\hline 14 & El precio de la tierra agrícola. & PTIERRA \\
\hline 15 & El costo de oportunidad de otros cultivos y pecuaria. & OTROCUL \\
\hline 16 & El desarrollo tecnológico del cultivo de la palta. & TECPALTA \\
\hline 17 & El desarrollo tecnológico en la industria de transformación de la palta. & TECIND \\
\hline 18 & La infraestructura de la distribución. & DISTRIB \\
\hline 19 & EI desarrollo del mercado de trabajo agrícola. & MERCTRAB \\
\hline 20 & Los impactos y limitaciones ambientales. & MEDIOAMB \\
\hline 21 & La política agraria. & AGRARIA \\
\hline 22 & El crecimiento del mercado de otros productos de la palta. & SUBPROD \\
\hline 29 & El aumento de la inversión extranjera directa del sector & IDE \\
\hline 24 & El tipo de cambio & CAMBIO \\
\hline 25 & La tasa de crecimiento de la economía peruana & CRESPERU \\
\hline 26 & La demanda internacional de la palta & DEMINPALTA \\
\hline
\end{tabular}

Fuente: MICMAC - Exportación y perspectiva del comercio de la palt Hass Peruana 


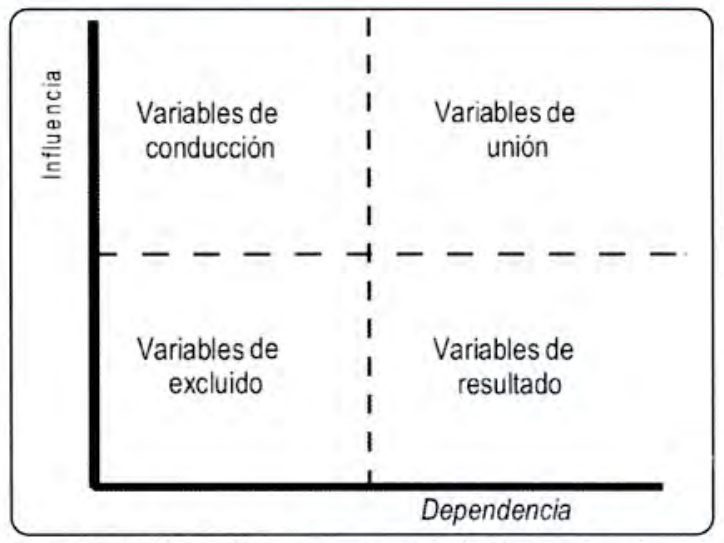

Gráfico $\mathrm{N}^{\circ}$ 01: Mapa motrocidad/ dependencia. Fuente : GODET, M. 2003, pág.119

La representación de los valores obtenidos para cada variable se encuentra en la Tabla $\mathrm{N}^{\circ} 05$ en la que vemos mejor el grado de influencia o conducir a una variable en el sistema y su grado de dependencia.

\section{Análisis de los actores clave involucrados en el sistema Mactor}

A partir del conjunto de variables descritas en el apartado anterior (con la posible excepción de la tasa de crecimiento de la economía mundial; variables que representan las capacidades y / o resultados del juego político), empezamos a analizar las estrategias de los principales actores que desempeñan un papel, utilizando el módulo MACTOR, que trabaja con el mismo algoritmo de operaciones con matrices.

Para simplificar la presentación de nuestro argumento, comenzamos por analizar los puntos fuertes relativos de los 16 jugadores seleccionados para el análisis.

Luego de construir una matriz cuadrada con estos dieciséis jugadores en filas y columnas se llenó valorando la influencia del actor " $\mathrm{i}$ " en el actor "j", según la siguiente escala:

\begin{tabular}{cll} 
Valor & & Nivel de Influencia \\
\hline $0:$ & & Sin influencia \\
1: & & Pequeña influencia \\
2: & & Medianas influencia \\
3: & & Fuerte influencia
\end{tabular}

El llenado de la matriz se realizó, pensando exclusivamente en el mercado actual y futuro de la palta Hass peruana y no una genérica influencia política o económica.

Se procedió a operar multiplicando las matrices de influencia dependiente por sí misma, hasta obtener la matriz de influencia directa e indirecta (MDII), que capta no sólo la influencia directa del actor "i" en el actor " $\mathrm{j}$ ", sino también su influencia a través de actores de terceros.

\section{Análisis del espectro de escenarios posibles Morphol}

Con base en el análisis de las variables de conducción y vinculantes se comenzó a construir algunos escenarios alternativos, sin dejar de producir una reducción en el número de variables más influyente y más grande en el mercado internacional de la palta producido en Perú.

Este proceso se realizó, en parte mentalmente,

Tabla ${ }^{\circ}$ 06: Mapa Motricidad Dependencia Indirecta del sector palta Hass en Perú

\begin{tabular}{|l|l|c|l|}
\hline $\mathbf{N}^{\circ}$ & \multicolumn{1}{|c|}{ Título largo } & Título corto & \multicolumn{1}{c|}{ Descripción } \\
\hline 1 & Organización Mundial del Comercio & OMC & Gestión del comercio mundial \\
\hline 2 & Gobierno de USA & USA & Administración Gubernamental \\
\hline 3 & Gobierno de la Unión Europea & UE & Administración Gubernamental \\
\hline 4 & Grupo de los países en desarrollo & G-20 & Gestión de producción y consumo \\
\hline 5 & Paises de África, Asia Pacifico y Caribe & AAC & Gestión de desarrollo \\
\hline 6 & Gobierno de Chile & CHILE & Administración Gubernamental \\
\hline 7 & Gobierno de Méjico & MÉJCO & Administración Gubernamental \\
\hline 8 & Asociación de Productores de palta Hass del Perú & PROHASS & Productores asociados de palta \\
\hline 9 & Comité de Palta Hass de Chile & HASSCHILE & Comité de productores \\
\hline 10 & Comunidad Andina de Naciones & CAN & Países de la Cuenca del Pacífico \\
\hline 11 & Asociación Sudafricana Productores de palta & ASPP & Productores asociados de palta \\
\hline 12 & Gobierno de Brasil & BRASIL & Administración Gubernamental \\
\hline 13 & Asociación Australiana Productores de Avocado & AAPA & Productores asociados de palta \\
\hline 14 & Gobierno de Perú & PERÚ & Administración Gubernamental \\
\hline 15 & Grupos Extranjeros con negocios en Perú & IDE & Grupo de inversores \\
\hline 16 & Gobierno de Indonesia & INDONESIA & Administración Gubernamental \\
\hline
\end{tabular}

Fuente: Mactor - exportación y perspectiva del comercio de la Palta Hass Peruana 
y en parte, con la ayuda de módulo Morphol (análisis morfológico).

En un primer intento, se ha levantado las nueve variables que se indica a continuación, en la que asumen dos o tres hipótesis de comportamiento para cada una de ellas.

a) Tasa de crecimiento de la Economía Mundial (TACREM).

b) Las controversias y escenarios de la hegemonía mundial (COESHM).

c) Capacidad de Intervención de los estados Nacionales y Políticas agrícolas Interna (CAINEN).

d) Los precios de la palta (PREPAL).

e) CAN y / o acuerdos con los Estados Unidos de América.(CAN).

f) Los acuerdos multilaterales en el ámbito de la OMC. Las políticas para el consumo de otras variedades de palta por razones de salud y economía(POLCON).

g) La competitividad de la palta en función de la tecnología y el precio de la tierra (COMPAL).

h) La producción de palta y los principales exportadores (PRPAPE).

Dos problemas de secuencia, tanto operacional como lógica, han surgido de esta primera aproximación. El primero fue la creación de más de 11 mil casos que, incluso, después de excluir combinaciones incompatibles, aún se conserva cinco mil escenarios posibles y, todavía, una serie de grandes escenarios con una probabilidad razonable. El segundo problema apareció en la definición del marco de hipótesis.

Con el análisis realizado, el conjunto de variables se redujo a menos de cinco, por el que se preparó un marco de supuestos que se pueden ver en la Tabla $\mathrm{N}^{\mathrm{o}}$ 07, con la probabilidad de ocurrencia dada por nosotros.

a) Tasa de crecimiento de la Economía Mundial (TACREM).

b)Las controversias y escenarios de la hegemonía mundial (COESHM).

c)Capacidad de Intervención de los estados Nacionales y Políticas agrícolas internacionales (CAINEN).

d)Los precios de la palta (PREPAL).

e) CAN y / o acuerdos con los Estados Unidos de América. (CAN).

Para toda economía mundial, las posibilidades de crecimiento corresponden a un escenario menos optimista a las proyecciones de la Organización de Cooperación y Desarrollo Económico (OCDE) que para el grupo de países que participan en la organización, y un escenario más optimista para la economía peruana, que acompañará al desarrollo mundial.

Las últimas políticas relacionadas con el consumo de otras variedades de palta presentan hipótesis con el 70\% (en general) y 30\% (restringido).

Una tabla de probabilidades permite el consumo de otras variedades existentes en la producción, compitiendo fuertemente con Hass, conocida en el mercado Internacional por sus características aceptadas por los consumidores.

\section{Evolución de la producción en cada uno de los escenarios}

\section{El multilateralismo y un crecimiento moderado}

La imagen representa la continuidad de las formas de regulación del comercio de bienes,

Tabla Nº7: Hipótesis Básicas para la Construcción de Escenarios.

\begin{tabular}{|c|c|c|c|c|}
\hline \multirow{2}{*}{ Dominio } & \multirow{2}{*}{ Variables } & \multicolumn{3}{|c|}{ Hipotesis } \\
\hline & & H1 & $\mathrm{He}$ & He \\
\hline \multirow{3}{*}{$\begin{array}{c}\text { Mercado } \\
\text { Internacional } \\
\text { de palta Hass } \\
\text { peruana }\end{array}$} & $\begin{array}{c}\text { Tasa de crecimiento de la } \\
\text { Economía Mundial }\end{array}$ & $\begin{array}{c}\text { Menos del } 2,5 \% \text { al año. } \\
50 \%\end{array}$ & $\begin{array}{l}\text { Más del } 2,5 \% \text { al af̃o. } \\
50 \%\end{array}$ & \\
\hline & $\begin{array}{l}\text { Las controversias y } \\
\text { escenarios de la } \\
\text { hegemonía mundial }\end{array}$ & $\begin{array}{l}\text { La continuidad de la } \\
\text { hegemonía mejicana. } \\
50 \%\end{array}$ & $\begin{array}{c}\text { Equilibrio de Méjico, el } \\
\text { bloque Chile, Colombia, } \\
\text { Perú y un bloque asiático. } \\
40 \%\end{array}$ & $\begin{array}{c}\text { La tensión y el conflicto } \\
\text { entre los polos de } \\
\text { producción. } \\
10 \%\end{array}$ \\
\hline & Los precios de la palta & $\begin{array}{c}\text { Menos de } \$ 1450 \text { por } \\
\text { tonelada. } \\
45 \%\end{array}$ & $\begin{array}{c}\text { Entre } \$ 1450 \text { y } \$ 1600 \text { por } \\
\text { tonelada. } \\
45 \%\end{array}$ & $\begin{array}{c}\text { Más de } \$ 1600 \text { por } \\
\text { tonelada. } \\
10 \%\end{array}$ \\
\hline
\end{tabular}

Fuente: MORPHOL Exportación y Perspectiva del Comercio de la palta Hass Peruana. 
especialmente agrícolas, por el predominio de la oposición a la reducción de los subsidios agrícolas y la liberalización del comercio de los actores más fuertes.

No hay onda proyectada más fuerte de liberalización que puede añadir un poco de estímulo adicional a la economía mundial, lo que significa pensar en una tasa más modesta de crecimiento, el 2,8\% anual de los últimos 20 años. En este caso (como en los dos siguientes), los países en desarrollo tendrán un crecimiento más rápido que el promedio de los últimos 20 años, lo que refleja la aceleración reciente de esta tasa y más en línea con las proyecciones de la Organización de Cooperación y Desarrollo Económico / FAO.

La principal limitación de la velocidad de este movimiento es la sustitución de un aumento de la capacidad de atracción de otras culturas y el precio del suelo, ya sea en Perú o en otros países, lo que puede compensar las ganancias de productividad a la competitividad de palta. Por lo tanto, creemos que el consumo de la palta seguirá creciendo en los próximos 20 años, depende de políticas explícitas para apoyar al productor $\mathrm{y} / \mathrm{o}$ consumidor.

Este movimiento de apreciación de la tierra, característica de la expansión del cultivo de palta en Perú incrementará la ocupación de tierras áridas con proyecciones de irrigación en la costa peruana.

\section{RESULTADOS}

\subsection{Evaluación Análisis Estructural Prospectivo Método Micmac}

Entre las otras variables clasificadas como de conducción, la política agraria aparece como una variable de baja dependencia dentro del sistema y con influencia moderada. Su influencia en el sistema no sólo puede ser importante en la producción, sino a la voluntad de los grupos extranjeros para invertir en la expansión del sector.

El siguiente paso del método de análisis estructural MICMAC consistió en multiplicar la matriz de influencia dependencia sucesiva por sí misma, de modo que se da cuenta de lo que debería tener cada variable a largo plazo.

El resultado obtenido muestra una homogeneidad relativa del sistema a largo plazo, con pequeños cambios en la posición de casi todas las variables. Entre las variables clasificadas como relevantes para el análisis, más que aumentar su influencia a largo plazo son las Controversias y Escenarios de la Hegemonía mundial y el Precio de la palta, entre las variables de conducción, la Organización Mundial del Comercio y La Política Nacional Agrícola vinculante variable de grupo. Aunque el desarrollo tecnológico del cultivo de la palta se mueva en el cuadrante superior izquierdo, pierde influencia en el sistema, lo que nos lleva a mantenerlo como una variable secundaria en el análisis.

\subsection{Análisis de los actores clave involucrados en el sistema Mactor}

El llenado de la matriz se asumió con la influencia de cada actor en los demás, pensando exclusivamente en el mercado actual y futuro de la palta Hass peruana y no una genérica influencia política o económica.

Tabla $\mathbf{N}^{\circ}$ 08: Supuestos demográficas y económicas 2012-2032.

\begin{tabular}{|l|c|c|c|c|}
\hline \multicolumn{1}{|c|}{ Rubro } & Escenario 1 & Escenario 2 & Escenario 3 & Escenario 4 \\
\hline Tasa de crecimiento del PIB & & & & \\
Economía Mundial & $2,3 \%$ & $3,0 \%$ & $2,4 \%$ & $2,0 \%$ \\
Los países desarrollados & $2,0 \%$ & $2,4 \%$ & $2,1 \%$ & $2,2 \%$ \\
Los países en vías de desarrollo & $3,5 \%$ & $4,3 \%$ & $4,0 \%$ & $4,1 \%$ \\
\hline Tasa de Crecimiento PoblacionalMundial & $1,1 \%$ & $1,1 \%$ & $1,1 \%$ & $1,1 \%$ \\
Los países desarrollados & $0,4 \%$ & $0,4 \%$ & $0,4 \%$ & $0,4 \%$ \\
Los países en vías de desarrollo & $1,3 \%$ & $1,3 \%$ & $1,3 \%$ & $1,3 \%$ \\
\hline Consumo de palta / Crecimiento del PIB & & & & \\
Los países desarrollados & $0,6 \%$ & $0,3 \%$ & $0,6 \%$ & $0,5 \%$ \\
Los países en vías de desarrollo & $0,1 \%$ & $0,05 \%$ & $0,05 \%$ & $0,1 \%$ \\
\hline Crecimiento consumo de palta per cápita & & & & \\
Los países desarrollados & $0,0 \%$ & $0,0 \%$ & $0,0 \%$ & $0,0 \%$ \\
Los países en vías de desarrollo & $0,5 \%$ & $1,0 \%$ & $0,5 \%$ & $0,5 \%$ \\
\hline
\end{tabular}

Fuentes: Organización de Cooperación y Desarrollo Económico, FAOSTAT y estimaciones del autor. 
Plano de influencias / dependencias directas

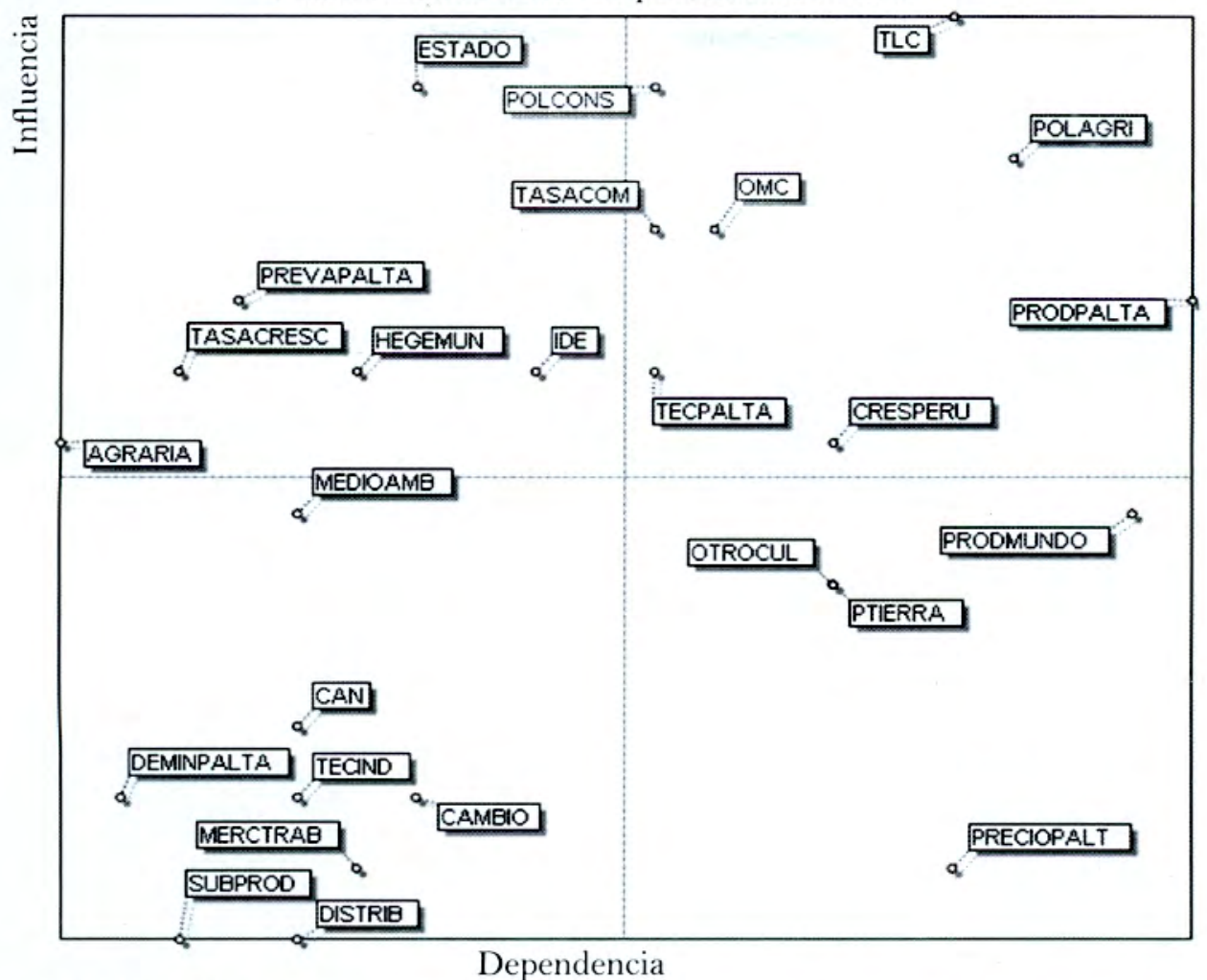

Gráfico $\mathrm{N}^{\circ}$ 02: Mapa motricidad dependencia directa del sector palta Hass en Perú. Fuente: MICMAC Exportaciòn y Perspectiva del Comercio de la palta Hass Peruana.

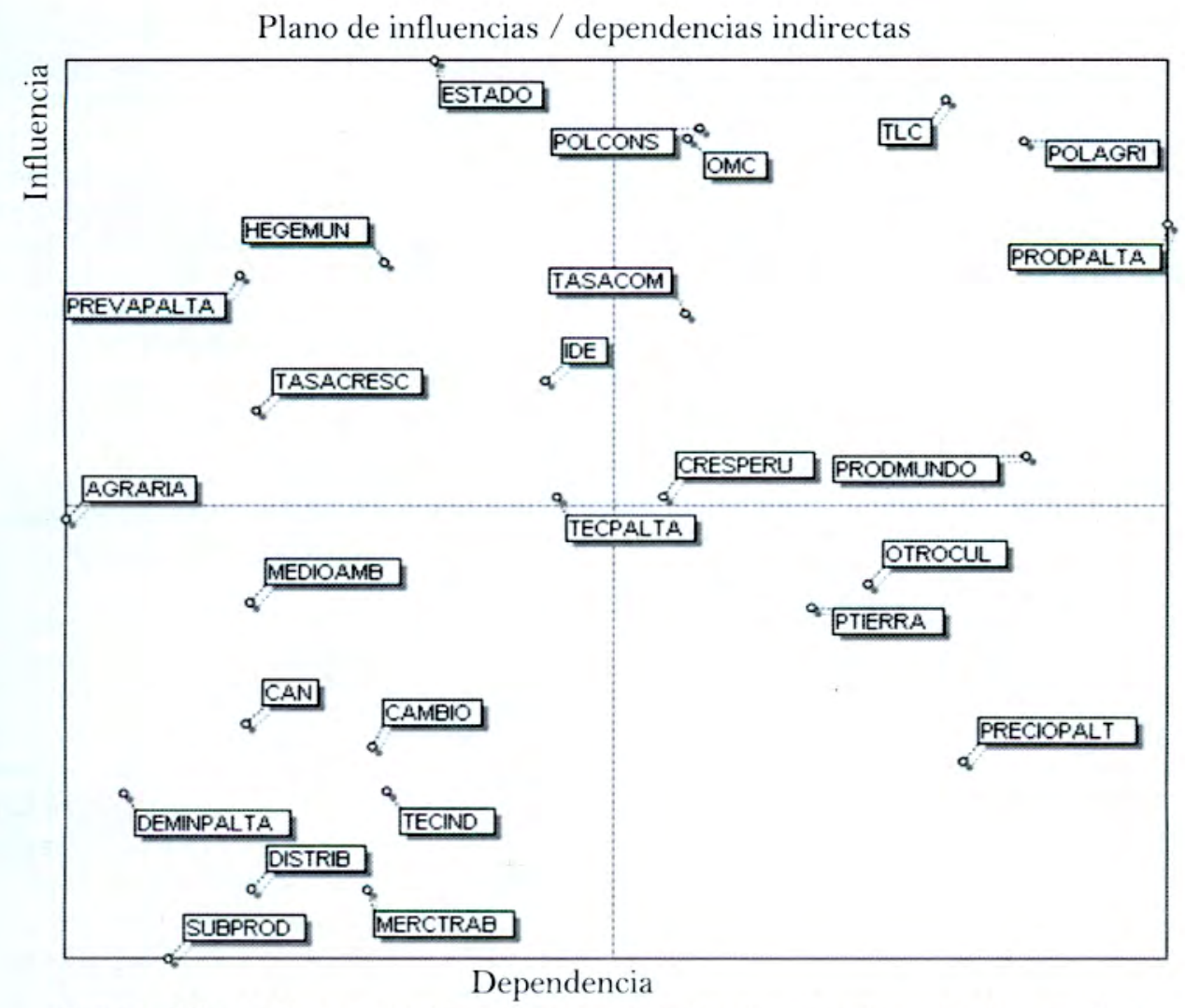

Gráfico $\mathrm{N}^{\circ}$ 03: Mapa motricidad dependencia indirecta del sector palta Hass en Perú. Fuente: MICMAC Exportaciòn y Perspectiva del Comercio de la palta Hass Peruana. 
Plano de influencias y dependencias entre actores

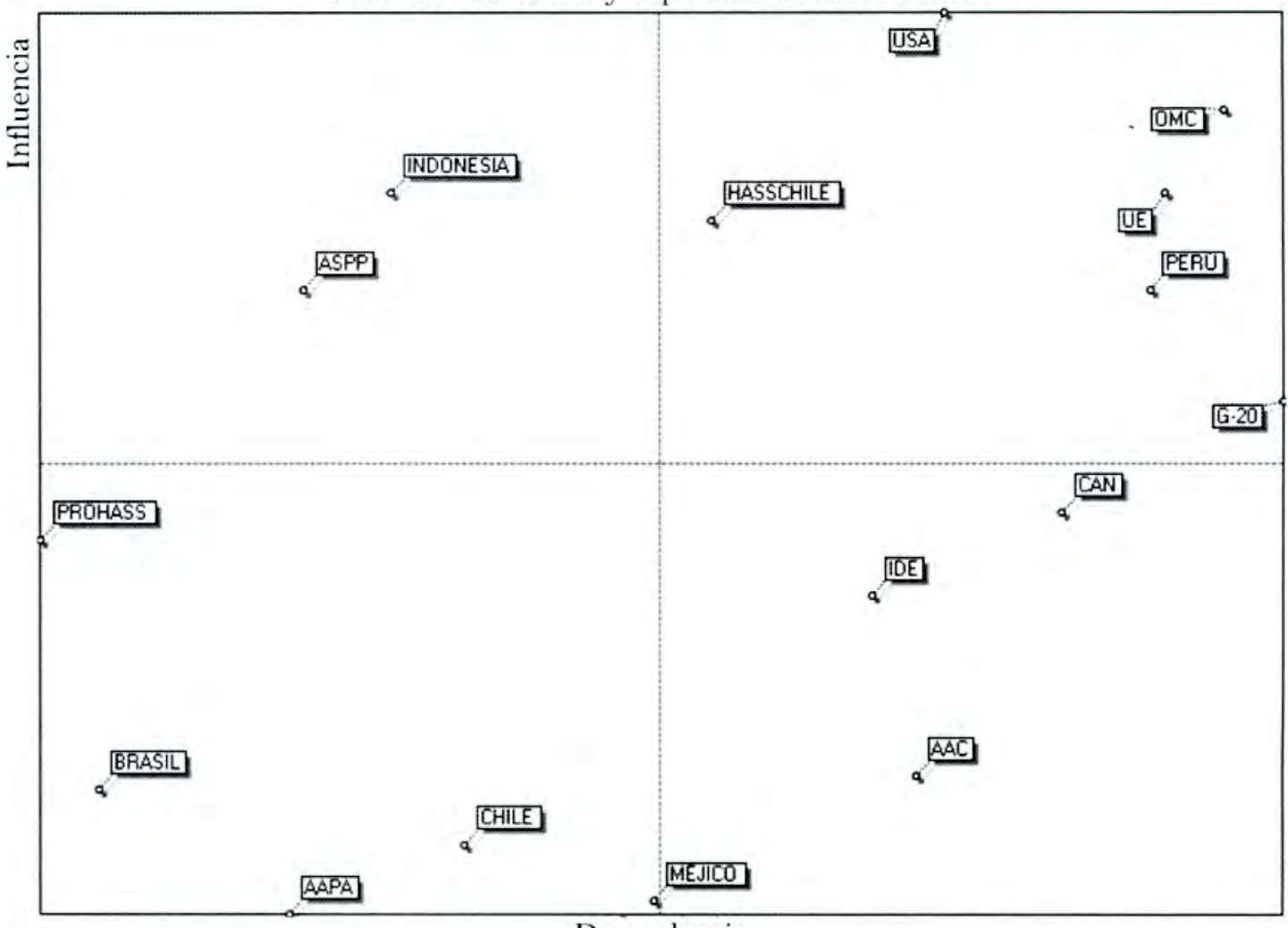

Dependencia

Gráfico $\mathrm{N}^{\circ}$ 04: Mapa Influencia Dependencia directos e indirectos de los actores.

Fuente : MACTOR exportación y perspectiva del comercio de la palta Hass Peruana

Otro indicador importante fue calculado por MACTOR por el coeficiente de competitividad de cada actor, que considera la influencia directa e indirecta del actor "i" (li) sobre la sumatoria de la influencia total, ajustado por su grado de dependencia (Di) en relación con los otros de la fórmula.

$$
\mathrm{Ri}=[(\mathrm{Ii}-(\mathrm{MDII}) \mathrm{ii}) / \mathrm{S}] \mathrm{x}[\mathrm{Ii} /(\mathrm{Ii}+\mathrm{Di})]
$$

Donde:

$$
\mathrm{S}=\mathrm{Si}=\mathrm{Si} \mathrm{Di} \mathrm{Ii}
$$

MDIIii efecto de retroalimentación:

$$
y=(i \rightarrow j \rightarrow i)
$$

En el Gráfico $\mathrm{N}^{\circ}$ 05, estos indicadores estándar muestran la competitividad de los dos actores. El hecho de que los dos no presentan alto grado de dependencia, al igual que otros actores más influyentes que ellos, los hace presentar como competitivos en nuestro sistema. Las organizaciones ecologistas asumen una posición similar, ya que su pequeña influencia se corresponde con una acción casi independiente de otros actores.

En este mapa se destacan el Gobierno de Indonesia y la Asociación Sudafricana, productores de palta como actores que, aunque no ejercen una fuerte influencia en la media o cualquiera de los otros actores del sistema, son influyentes tanto por su influencia indirecta a través de un gran número de otros actores como por su bajo dependencia de los demás. Estados Unidos de América es el mayor importador de palta en el mercado. En cuanto a la Unión Europea, en virtud de la posición que goza, no se ve sustancialmente afectada por las políticas económicas en la alimentación, ya que otras variedades de palta alternativos seguirán siendo complementarios a la variedad Hass en la matriz de alimentos vegetales.

A partir de las similitudes y diferencias de cada par de actores para cada objetivo, MACTOR construye convergencias matrices (CAA) y divergencias (DAA), con la que produce un mapa de las distancias entre los actores respecto a los objetivos, como podemos ver en la Gráfico $\mathrm{N}^{\circ} \mathrm{O}$.

El cuadrante superior derecho de la Gráfica $\mathrm{N}^{\circ}$ 04 muestra los actores que tienen influencia en el sistema. Ellos están sujetos a un grado razonable de la dependencia de la acción de otros agentes, con la posible excepción del Comité de palta Hass de Chile que se ve favorecida por una dependencia mucho menor que el de otros actores. En esta figura, vemos que el Gobierno de Perú tiene una influencia razonable en el sistema, aunque inferior 
Histograma de relaciones de fuerza MDI

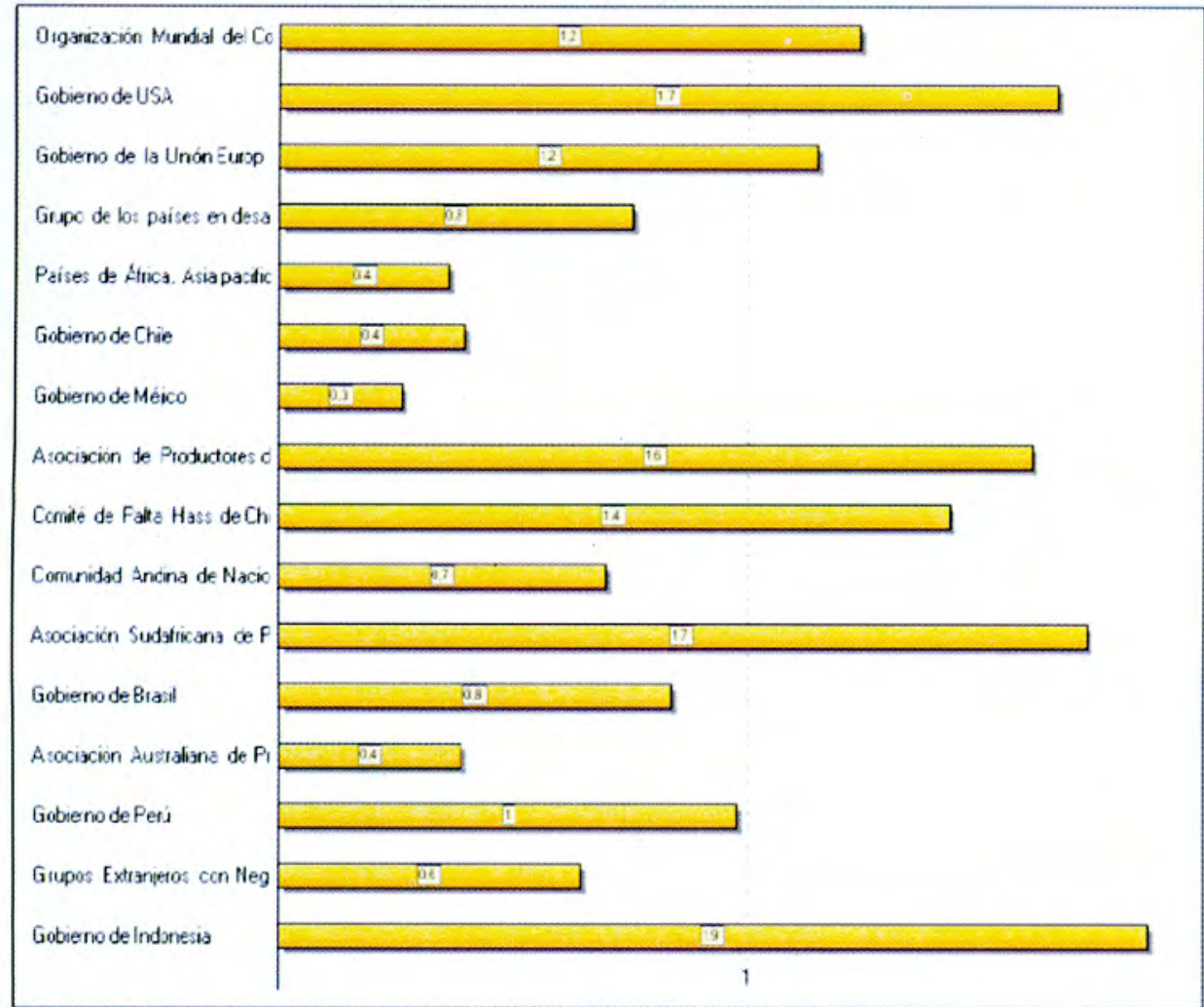

Gráfico $\mathbf{N}^{\circ}$ 05: La competitividad de Actores por la influencia directa-indirecta.

Fuente : MACTOR exportación y perspectiva del comercio de la palta Hass Peruana.

Plano de distancias netas entre actores

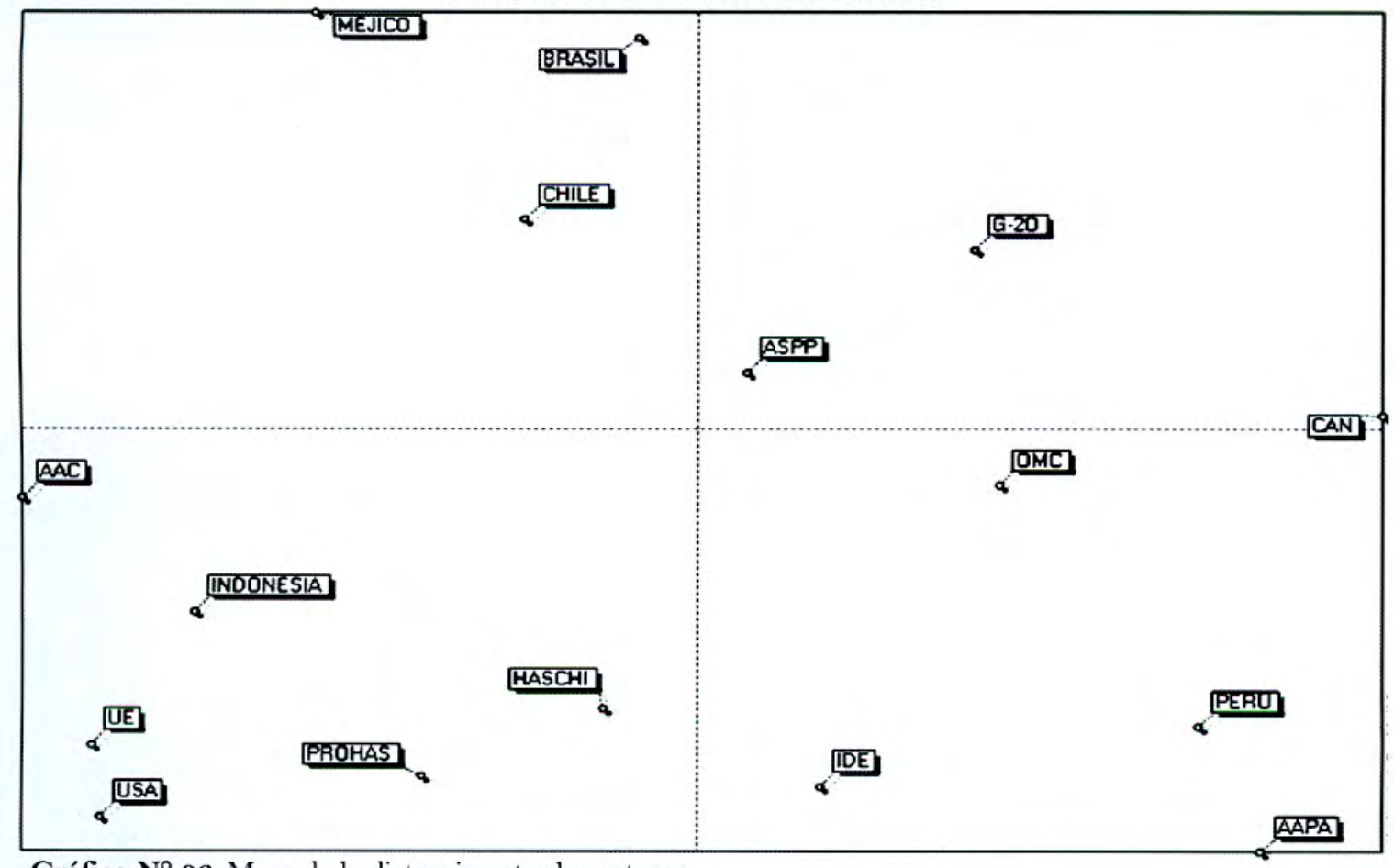

Gráfico $\mathbf{N}^{\circ}$ 06: Mapa de la distancia entre los actores

Fuente: MACTOR exportación y perspectiva del comercio de la palta Hass Peruana 
Tabla $\mathrm{N}^{\circ}$ 08: Mapa de influencia dependencia directa e indirecta entre actores

\begin{tabular}{|l|c|c|c|c|c|c|c|c|c|c|c|c|}
\hline & REDSU & SECAPR & PRPRIN & PRETAR & RELIPR & PRSAAG & MAPRPA & MAPRPR & REBAPA & LIFLCA & Suma absoluta \\
\hline OMC & 1 & 0 & -1 & 0 & 0 & 0 & -1 & -1 & 1 & 1 & 6 \\
\hline USA & -1 & 1 & 1 & 1 & 1 & 1 & 0 & 1 & -1 & 1 & 9 \\
\hline UE & -1 & 1 & 1 & 1 & 1 & 1 & -1 & 1 & -1 & 1 & 10 \\
\hline G-20 & 1 & 1 & 0 & 0 & 0 & -1 & -1 & 1 & 0 & -1 & 6 \\
\hline AAC & -1 & 0 & 1 & 1 & 0 & 1 & 0 & 0 & -1 & 0 & 5 \\
\hline CHILE & 0 & 1 & 1 & 0 & -1 & 0 & -1 & 0 & 0 & 0 & 4 \\
\hline MÉJICO & 0 & 0 & 1 & 1 & -1 & -1 & -1 & 0 & -1 & 0 & 6 \\
\hline PROHASS & 0 & 0 & 1 & 0 & 1 & 1 & 0 & 0 & 0 & 0 & 3 \\
\hline HASSCHILE & 0 & 1 & 0 & 0 & 1 & 0 & -1 & 0 & 0 & 1 & 4 \\
\hline CAN & 1 & 1 & -1 & -1 & 0 & -1 & 0 & 1 & 1 & -1 & 8 \\
\hline ASPP & 0 & 1 & 0 & 0 & -1 & 0 & 1 & 0 & 0 & 0 & 3 \\
\hline BRASIL & 0 & 1 & 0 & 0 & 0 & -1 & -1 & -1 & -1 & -1 & 6 \\
\hline AAPA & 1 & 1 & -1 & -1 & 1 & 0 & 1 & 1 & 1 & 0 & 8 \\
\hline PERÚ & 1 & 1 & -1 & -1 & 1 & 0 & -1 & 1 & 1 & 0 & 8 \\
\hline IDE & 0 & 1 & 0 & 0 & 0 & 0 & 0 & 1 & 1 & 1 & 4 \\
\hline INDONESIA & -1 & 1 & 1 & 0 & 1 & 0 & -1 & 0 & -1 & 1 & 7 \\
\hline Número de acuerdos & 5 & 12 & 7 & 4 & 7 & 4 & 2 & 7 & 5 & 6 & \\
\hline Número de desacuerdos & -4 & 0 & -4 & -3 & -3 & -4 & -9 & -2 & -6 & -3 & \\
\hline Número de posiciones & 9 & 12 & 11 & 7 & 10 & 8 & 11 & 9 & 11 & 9 & \\
\hline
\end{tabular}

a la de los gobiernos de los Estados Unidos de América, la Unión Europea y la Organización Mundial del Comercio.

El siguiente paso del análisis fue para seleccionar un conjunto de objetivos, tratando de mantener la máxima independencia posible entre ellos, y llenar la matriz (2MAO, que se puede ver en la parte central de la Tabla $\mathrm{N}^{\circ} \mathrm{O}$ ), que relaciona la posición de cada actor "i" con cada objetivo "j". Esta operación permitió a una nueva valoración de los jugadores al incorporar el análisis de las convergencias y divergencias entre cada uno, abriendo la posibilidad de alianzas entre explícito o no un subconjunto de actores.

En primer lugar dos observaciones importantes surgen de la lectura de la matriz. Por el lado de los objetivos, la seguridad de la calidad de producción se presenta como un objetivo perseguido por la mayoría de los actores, sin la oposición de ninguno de ellos. Aunque se trata de un objetivo genérico (que reduce la aparición de controversias), muestra un campo de convergencia que se está trabajando por actores que buscan aumentar la participación de la producción peruana en el comercio mundial, en particular el Gobierno de Perú.

Por el lado de los actores, es importante tener en cuenta las organizaciones. Así las Asociación de productores de palta Hass del Perú y el Comité de palta Hass de Chile, que son fuertes en su relación con los demás y tienen poca participación con la lista de los objetivos planteados, lo que reduce su papel en el análisis de las consecuencias de sus acciones en un enfoque reducido de objetivos.

$\mathrm{El}$ análisis de los objetivos se puede mejorar con la multiplicación de los actores metas por el grado de competitividad de cada actor (Ri), y tendrá en cuenta la posición de un actor por un objetivo político. A continuación presentamos el histograma de la movilización de los actores para cada objetivo, el cual permite una mejor visualización de los resultados.

La seguridad en la calidad de producción debe asumir el papel de la convergencia en la acción política de los actores, que deben seguir una fuerte presión ambiental al reducir el empleo de fertilizantes, que apunta a un escenario favorable para la expansión del mercado de productos ecológicos.

A partir de las similitudes y diferencias de cada par de actores para cada objetivo, MACTOR construye la convergencia de matrices (CAA) y divergencia (DAA), con la que produce un mapa de las distancias entre los actores respecto a los objetivos, podemos ver en la Gráfico 07 .

La lectura de este mapa confirma algunas hipótesis iniciales, y trae nueva información. Como 
Plano de distancias netas entre caracteres

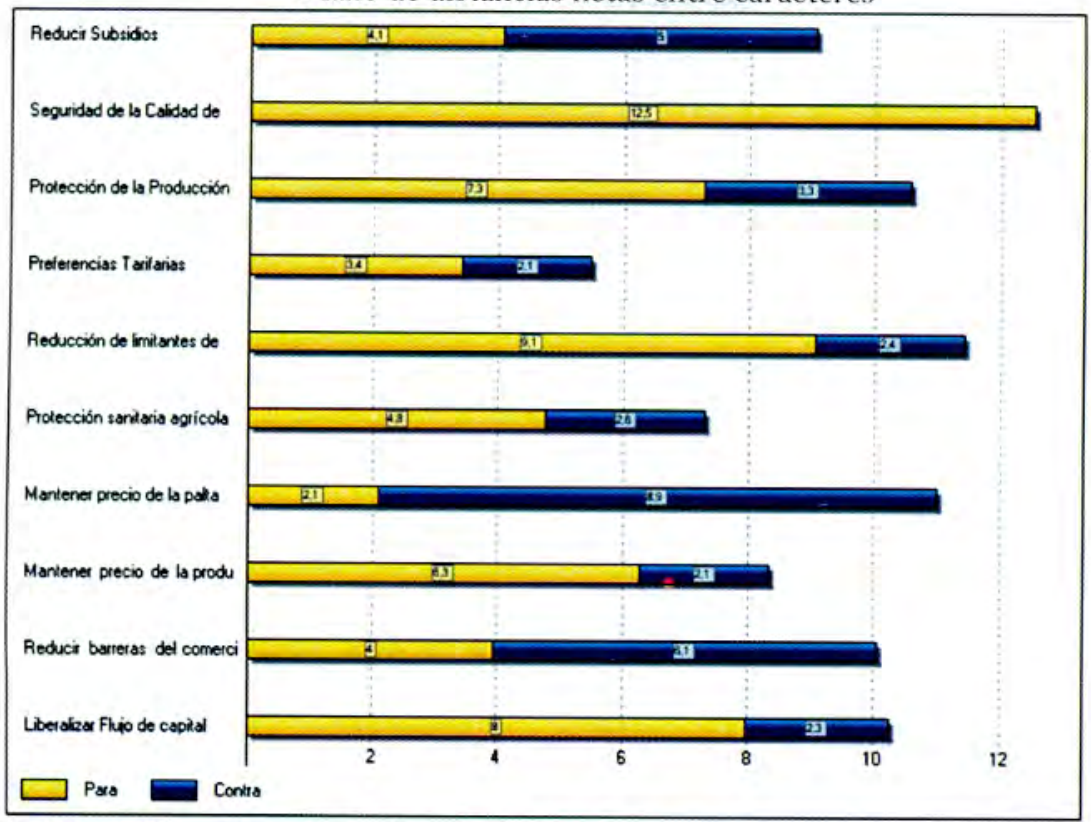

Gráfico $\mathrm{N}^{\circ}$ 07: Mapa de la distancia entre los actores.

Fuente : MACTOR exportación y perspectiva del comercio de la palta Hass Peruana.

era de esperar, los gobiernos de los Estados Unidos de América y la Unión Europea están muy cerca, lo que apunta a alianzas tácitas o no, en los debates sobre el futuro de la demanda de palta. La nueva información del mapa de distancias es que el Gobierno de Perú está alejado, tanto de la Comunidad Andina de Naciones y del Grupo de los países en desarrollo, lo que indica que los conflictos no siempre serán a través de una alianza comercial y productivo con estos actores.

Se muestra una cercanía mucho mayor con los objetivos de la Organización Mundial del Comercio, por lo que suponemos que este es el aliado natural de los productores peruanos de palta.

\subsection{Análisis del espectro de escenarios posibles.}

El mercado mundial de la palta espera aumentar sólo en términos de crecimiento de la población que consume vegetales y hortalizas, tanto en los países desarrollados y Perú, por el hecho de que estos mercados ya están saturados. Los demás países presentan un crecimiento en los niveles per cápita de consumo indicados en la Tabla $\mathrm{N}^{\circ} 09$, ajustado por la tasa de crecimiento de sus economías.

En este escenario, se supone que la palta producido en Perú seguirá representando un $6,65 \%$ del consumo mundial.
Tabla $\mathrm{N}^{\circ}$ 09: Consumo y exportación Peruana de Palta.

\begin{tabular}{|l|r|r|r|}
\hline \multicolumn{1}{|c|}{ Toneladas de palta } & \multicolumn{1}{c|}{$\mathbf{2 . 0 1 2}$} & \multicolumn{1}{c|}{$\mathbf{2 . 0 2 2}$} & \multicolumn{1}{c|}{$\mathbf{2 . 0 3 2}$} \\
\hline Consumo de palta & 883944 & 1590019 & 2862035 \\
\hline Países desarrollados & 574.174 & 985812 & 1717221 \\
\hline Países en desarrollo & 309170 & 604207 & 1144814 \\
\hline Perú & 17850 & 24990 & 34986 \\
\hline Exportaciones Peruanas & 83947 & 116686 & 163960 \\
\hline
\end{tabular}

Fuente: FAOSTAT, COMTRADE y proyecciones de la investigación.

Una característica importante de los escenarios es que la expansión de la producción tiene lugar dentro de un marco regulador y un entorno económico muy diferente de la que existía durante la década 1990.

En la actualidad la inversión está siendo financiado por fuentes de bancos nacionales y entidades financieras del país. Se ve el crecimiento del sector agrícola por la expansión del cultivo para la exportación.

Tabla $\mathbf{N}^{\circ}$ 10: El consumo y las exportaciones de la palta de Perú.

\begin{tabular}{|l|r|r|r|}
\hline \multicolumn{1}{|c|}{ En toneladas de palta } & \multicolumn{1}{c|}{$\mathbf{2 . 0 1 2}$} & \multicolumn{1}{c|}{$\mathbf{2 . 0 2 2}$} & \multicolumn{1}{c|}{$\mathbf{2 . 0 3 2}$} \\
\hline Perú & 17850 & 24990 & 34.986 \\
\hline Exportaciones Peruanas & 83947 & 116686 & 163960 \\
\hline Palta adicional requerida & & 40479 & 56670 \\
\hline Área adicional requerida (ha) & & 4400 & 6160 \\
\hline
\end{tabular}

Fuente: Generada por MACTOR, información del autor. 
El crecimiento de la producción se ubicará en la región norte del país: La Libertad, Lambayeque y Piura, ampliando las áreas agrícolas y agropecuarias en buenas condiciones para el uso de equipos mecanizados, aunque su crecimiento demandará mayor consumo de agua. Estas regiones cuentan con otros tipos de cultivos como: limón, mango, caña de azúcar que pueden absorber parte del área de cultivo.

Una característica importante de estos escenarios es que la expansión de la producción tienen lugar dentro de un marco legal de inversión en el país. En la actualidad, la inversión está siendo financiada por los bancos y entidades crediticias. Significa que el sector debería intensificar su concentración empresarial con un mayor grado de internacionalización de la producción, en el interior y los grupos nacionales más fuertes. Por otra parte, no parece el aumento de la propiedad extranjera en la producción agrícola y del sector agroindustrial.

\section{Escenario: Bloque Hemisférico}

Este escenario, con una menor probabilidad de ocurrencia, implica reorientar la dinámica expansión del sector agroindustrial en el Perú. Los mercados europeos y asiáticos tienden a limitar (pero no eliminar) la exportación peruana, ocupada por los productores intrabloque (principalmente centroamericano y africano). Las otras transacciones en el mercado de los Estados Unidos de América intensificaría (con o sin CAN) las exportaciones de palta.

Todas las proyecciones de consumo de la palta son los mismos en el escenario multilateral, con un crecimiento moderado, ya que la tasa de crecimiento de la economía mundial y las políticas de consumo de otras variedades de palta son equivalentes. Este cambio tiene un gran impacto en las exportaciones peruanas.

\section{Escenario: Disputa hegemónica}

Este es un escenario muy extremo, con un aumento de los conflictos localizados como resultado de la competencia por la hegemonía, el crecimiento promedio de la economía mundial sería aún más baja que en los otros escenarios ( $1,1 \%$ anual), y un mercado menos flotante para los productos agrícolas.

El mercado de la palta se vería afectada sólo por la desaceleración del crecimiento económico de los países en desarrollo, una proporción menor de su importancia en la canasta básica de la población de menores ingresos.
Sin embargo, los productores peruanos perderían una parte del mercado internacional por el auge del proteccionismo entre bloques y países. En la Tabla $\mathrm{N}^{\circ} 10$, vemos el efecto de estos supuestos en el mercado global, el supuesto de que las exportaciones peruanas de palta se reduciría en un $2,5 \%$.

\section{Futuro de la producción de palta en la economía peruana.}

Como hemos visto los resultados obtenidos en las proyecciones de producción y en el área plantada de palta, se espera una rápida expansión en los próximos 10 años, al menos en los primeros tres escenarios. Sin embargo, este crecimiento se ha enfriado en el mediano plazo, manteniéndose por debajo de la evolución del PIB peruana en los supuestos anotados en los escenarios. El multilateralismo, el crecimiento moderado y el Bloque Hemisférico, en este último caso, tendría mayor rapidez por medio de crecimiento optimista $5 \%$ por año.

Este comportamiento es consistente con las características de los mercados enfocados en este sector. En cuanto se refiere a palta, se trata de un producto con baja elasticidad-ingreso de los países desarrollados y también en el mercado peruano, que ya ha alcanzado un nivel de consumo per cápita cercano a su saturación. Todavía hay espacio para el crecimiento del consumo en los países en desarrollo, pero la premisa de mantener la participación actual de la producción peruana en el suministro global ya es bastante optimista.

El mercado para la palta tiene una expectativa de crecimiento para los próximos 10 años, por otra parte, debe seguir el crecimiento de otras variedades de palta. Los continuos avances en la tecnología y, sobre todo, la necesidad de producción ecológica debería hacer que esta tendencia continuara en el futuro, lo que se traduce en mayores tasas de consumo de diversas variedades de palta.

\section{CONCLUSIONES}

- El crecimiento de la demanda en los próximos diez años, sin duda, va a suponer un cambio en la estructura productiva del sector agrícola. A nuestro juicio, la rapidez con que se esperan los nuevos cambios, incluye la posibilidad de grupos nacionales que retornan del exterior inviertan en el sector.

- Es necesario nuevas áreas de producción para afrontar el incremento de la demanda. En nuestra opinión, la política de Estado tiene 
como eje temático la competitividad del país, por lo tanto, deberá ampliar la frontera agrícola, de hecho, los productores tendrían grandes ventajas con el empleo de la buenas prácticas agrícolas.

- Considerando que el sector espera duplicar su producción en los próximos 20 años y que la expansión de frontera agrícola, será en la región norte del país: La Libertad, Lambayeque y Piura. Se esperan cambios muy fuertes en los mercados regionales.

- Por lo tanto, dicha expansión traerá un impacto sustancial en la población de la zona relacionado con el mercado laboral y las migraciones sociales estacionales.

\section{REFERENCIAS BIBLIOGRÁFICAS}

Superintendencia Aduanas Nacional (2011). Anuario estadístico de exportación del Perú. Lima.

Cereceda Meneses, Carlos (2010) Métodos y técnicas de la Prospectiva. Editorial Lirano S. A., Madrid, España.

Lerma Kirchner, Alejandro (2000). Comercio Internacional, Metodología para la formulación de estudios de competitividad empresarial, Ediciones, contables, administrativas y fiscales S. A, C. V. Méjico.
Godet, Michel, ARCADE Jacques, y otros (2004). Análisis Estructural con el Método Micmac, y Estrategia de los Actores con el Método Mactor. Millennium Project del American Council for the United Nations University, Washington, USA.

Ruibal Handabaca, Alberto (2006). Corredores Interoceánicos suramericanos, Editorial Fimart S.A.C., Lima, Perú.

Soms García, Esteban (2002). Estrategias y planes regionales, Guía Metodológica Editorial Lirano S. A., Madrid, España.

Sainz de Vicuña Ancin, José María (2011). Plan de Marketing en la práctica, Editorial ESIC., Madrid, España.

Villajuana Pablo, Carlos (2006). Gestión Estratégica Integral, Editorial Editora Cartolan E.I.R.L., $3^{\text {a }}$ edición. Lima, Perú.

Villajuana Pablo, Carlos y Ríos, Jonatan (2008). Gestión Estratégica Publica, Editorial Ediciones Villajuana consultores S.A.C., Lima, Perú.

\section{Correspondencia:}

Bartolomé Anyosa Gutierrez

Ciudad Universitaria fundo "Los Granados" Av. Miraflores s/n Tacna - Perú

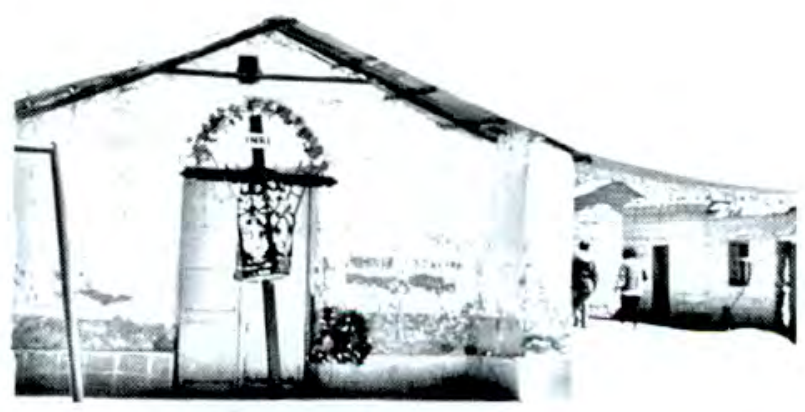

Capilla de Huaytire 